\title{
Research on Improvisational Accompaniment Training and Practical Teaching in Piano Teaching
}

\section{Fanxing Yang}

Zhaotong University, Zhaotong, 657000, China

\begin{abstract}
Keywords: piano teaching; improvisational accompaniment training; practical teaching; research
\end{abstract}
\begin{abstract}
In the process of vocal performance, piano accompaniment is one of the important musical instruments, and improvisational accompaniment is very important. At the same time, it also requires the piano performer to play the appropriate tune in combination with the performance scene. Therefore, in the process of piano teaching, it is necessary to strengthen the training, which in turn can promote the piano performer to fully exert his comprehensive role in the performance. In this paper, the piano teaching method is mainly used to analyze the methods of improvisational accompaniment training and the specific teaching methods. It is hoped that the improvisational accompaniment ability of the piano performers can be effectively improved. At the same time, the instructors can also bring a certain reference.
\end{abstract}

\section{Introduction}

Vocal arts can play an active role in the performance. Among them, piano accompaniment is loved by people as an inseparable part of the musical performance. Many excellent Chinese and foreign song arts use the piano as an important accompaniment basis. In the piano teaching process, teachers need to strengthen the guidance of the piano learners and can help the piano performers play the appropriate tunes in combination with specific situations. Therefore, this requires that the performer can combine the characteristics of the piano and the performance of the work to create, so that the improvisation can bring people a good sense of hearing. In the following, the specific training methods and practical teaching of improvisational accompaniment are mainly analyzed, and it is expected that they can serve piano learners well.

\section{Master the Basics of Playing Skills}

The difference between improvisational accompaniment and piano performance is mainly that the entire accompaniment performance must be effectively required for singing at the time of service. Therefore, on the basis of the preparation of the basic melody, harmony knowledge and assignment theory are also required to guide the improvisation. Playing can show the comprehensive effect of the accompaniment texture, and at the same time, it can effectively adjust the overall effect of the entire piano shift. During the piano training process, the performer is required to master the keyboard [1], and at the same time, it is also possible to skillfully play different syllables, chord connections, and arpeggio and chord connecting sounds, and then it is possible to carry out rapid transposition in conjunction with the song situation. It can be seen that impromptu accompaniment is a highly difficult performance technique, which requires the performers to undergo long-term tempering, and at the same time can practice repeatedly in the piano practice teaching process, laying a solid foundation for improvisation accompaniment.

\section{Strengthen Harmony Arrangements}

The core part of piano impromptu accompaniment is harmonization. In order to enhance the quality of piano accompaniment, learners need to strengthen the learning of harmonic knowledge, and then can arrange a good accompaniment for different songs to perform. 


\subsection{Pay Attention to Harmony Design.}

In piano performances, improvisational accompaniment requires the strengthening of harmony design. This is the "soul" of improvisational piano accompaniment. However, it is necessary to meet this requirement. Piano learners need to combine the understanding of melody knowledge to perform harmonistic design, and thus better. Meet people's requirements for piano appreciation. First, we must fully design the harmony design at the beginning of the song and the climax. At the same time, we must pay attention to the harmony and functionality of the harmony [2] so that the piano performance can better deal with semi-termination and termination. Second, configure other chords according to the rules and effects of the harmony compilation to effectively improve the expression of the songs. Therefore, in the piano teaching process, teachers need to combine the theoretical knowledge of harmony with practice, and then complete the training requirements with good chord knowledge. The learners need to focus on the training process, in which harmony theory knowledge is the foundation, the learners need to play solid basic knowledge, and then in the training process also need to tie chord skills, can combine keyboard and harmony knowledge for comprehensive training [3]. In addition, the learner also needs to strengthen the training of chord and chord connection skills, which in turn can enhance the acoustic perception of the piano and can be used in the corresponding songs to provide good help for the learners in improvising accompaniment performances.

\subsection{Pay Attention to the Use of Polytactics.}

In the process of improvising accompaniment, the piano needs to be integrated with different melody, song characteristics, and singing objects. Under normal circumstances, lyrical melody and artistic melody as the basic methods of accompaniment, in the song performance can increase the color [4]. Therefore, piano learners need to apply some polyphony techniques properly so that the songs can be better processed. In this way, the artistic performance of musical accompaniment can be effectively enhanced. In the daily training process, learners need to absorb a lot of artistic melody, and can fully display the charm of the accompaniment according to the requirements of different singers, so that singing and interval can be performed in a timely manner through the piano accompaniment, in the complex tactics Shows the artistic value of singing [5].

\section{The Expression Way of Designing Accompaniment Sound Type}

In an impromptu accompaniment, the accompaniment pattern is a very critical step. During the accompaniment performance, the learner needs to design an appropriate accompaniment texture according to the specific content of the melody and emotions. This part is the key to improvisation accompaniment, in order to be able to be better To achieve a good performance, the learner needs to master the style of good accompaniment, and at the same time, it can combine various emotions to design the corresponding accompaniment texture [7]. In the process of piano accompaniment, there are several types of accompaniment sounds:

In the improvisation accompaniment is one of the important ways of rhythmically changing the chords. Normally it is necessary to use the chord as a bass and place it in a strong beat, and each part is mainly placed on a weak beat, which makes the accompaniment possible. In addition, it is necessary to master certain techniques in the training process, which can make the entire piano accompaniment to achieve good results, and then give people a sense of vitality. The accompaniment can be used in music such as marching, waltzing, and queuing songs.

In the piano accompaniment, some intensive chords are mainly used as the background melody of the entire song. Through the method of harmony rhythm, a slow voice can be fully expressed, and at the same time, the vocal part can have good stability. The accompaniment can produce a quiet and stable, majestic, tragic and innocent simple effect.

In the piano accompaniment, the chords are mainly processed as an important teaching form, and can be played in a tone-by-tone manner to form an integrated texture of the monophonic flow performance music. The performances of the performance include: gentleness, exquisiteness, and 
gracefulness. As well as lightness, twisting, and long [8]. The use of this method in impromptu accompaniment is mainly aimed at some lyrical song performances, which can better enhance the appeal of the songs.

In the piano accompaniment, by decomposing the chord type, the entire harmony rhythm pattern can be combined to form a mixed type sound pattern. At the same time, this method can also reduce some mechanical rhythm, and can also make the accompaniment. Some of them have good melody and fluidity. Therefore, in order to enhance the effect of the entire accompaniment, the accompaniment is elegant and affectionate, full of poetry and delicate and beautiful effects [9]. The accompaniment is often used in lyric solo songs.

In explaining the relevant knowledge, the teacher needs to instruct the students to decompose the chords. At the same time, some extra sounds must be added to the strings to further optimize the legalization process. Decomposing the corresponding chord pattern by means of a sound pattern can better promote the entire accompaniment to be more flexible and changeable. In addition, through the presence of non-melody accompaniment textures, the accompaniment can be better presented, making the whole melody more lively and free.

In the process of decomposing chords, the two octaves or above are mainly subjected to continuous decomposition, because the processing method is mainly focused on chords, and sometimes other chords can be added. However, there are still limitations in the use of this type of sound. In the performance, it is not possible to use the method of the whole song. In the accompaniment, it is mainly used in the climax position or the end of the music [10]. In the bass area, an open arrangement method is used, and in the middle area and the high range area, the dense arrangement method is mainly used. In accompaniment performances, this type of sound is often left-handed, and the two-handed arpeggios also have good results, namely: enthusiasm, imposing, magnificent, passionate, and simple and smooth.

\section{Analyze Songs and Design Accompaniment}

Because the content of different songs is different, these contents are not only reflected in the lyrics, but also reflected in the melody, so in the accompaniment you need to strengthen the analysis of the song's genre, expressions, and musical forms, etc., and then make the song content Can improve the overall effect in good accompaniment. In the improvisational accompaniment process, the corresponding song theme and genre are first determined through familiar melody and lyrics, and then the factors such as the style, structure, beat, and speed of the corresponding song are fully analyzed, and the arrangement and harmonization can be performed in a good manner. In the training process, learners are required to pay attention to the analysis of the content of the songs and to provide their own understanding before they can improvise the songs.

\section{Enhance Comprehensive Capabilities}

In the process of improvising piano accompaniment, accompaniers need to have a good comprehensive ability. Not only do they need to master the skills of solid piano playing, they also require the accompaniment to have a good psychological quality. During the training process, learners need to consciously adjust their own mental state while mastering basic accompaniment knowledge, and prevent the occurrence of some unpleasant conditions in the accompaniment, such as the situation of full body tension or finger trembling. In addition, learners also need to improve their confidence and gradually accumulate experience in good accompaniment performances. From the practice of accompaniment, we can see that there are various situations. For example, some singers suffer from unstable rhythm and some appear to run away. All of these require the accompaniment to have good comprehensive ability and encounter bad experiences. Under the circumstances, the integrity of the music performance can also be fully guaranteed. Again, in the accompaniment process of the soloist, if the soloist suffers from poor pitching, the accompanist will need to use the correct pitch and rhythm to drive the singer and help the soloist to successfully complete the performance. 


\section{Conclusion}

Improvisation accompaniment is one of the important forms of piano performance in piano performance. This performance not only reflects the performer's flexibility and quick thinking, but also requires the performer to be proficient in the relevant acoustic assignments. Knowledge, in conjunction with the combination of keys, correctly pops up the corresponding song, giving people a good perspective to enjoy. In the future piano teaching process, it is necessary to strengthen improvisational accompaniment training, and at the same time, gradually improve the ability of improvising accompaniment in combination with the practice of piano performances.

\section{References}

[1] Chen Ke. Research on the Application of Overturning Classroom Teaching in Piano Teaching in Higher Vocational Colleges [J]. Popular Literature, 2017, 90(23): 224-225.

[2] Li Lina. A tentative analysis of the ways to develop students' musical sensation in piano teaching [J]. Journal of Changchun Normal University, 2017, 36(12):161-162+165.

[3] Xia Yan. Application of Orff Teaching Method in Piano Teaching[J]. Art Review, 2017, 23(23): 117-118+133.

[4] Sun Shanshan. The construction and thinking of the innovative model of piano teaching [J]. Northern Literature (late), 2017, 12(08):196.

[5] Huang Yaxi. Exploration of Piano Teaching Method Based on Psychological Development of Preschool Children[J]. Journal of Ningbo Institute of Education, 2017, 19(02): 6-9+20.

[6] Zhang Lihong. Traditional Piano and Applied Piano: An Integrated Teaching Approach to Piano for Integrated Music Education in Universities [J]. Art Research, 2017, 02(04): 127-129.

[7] Yu Lin, Zhang Yinghui. The Application of Orff's Music Education Method in Piano Teaching of Higher Vocational Education [J]. Journal of Yanbian Education College, 2017, 31(01): 78-80+83.

[8] Huo Shuxia. On the Cultivation of Creative Thinking in Piano Teaching: A Review of "A New Approach to Piano Teaching" [J]. Chinese Journal of Education, 2017(09):144.

[9] Wu Hao. Research on the Construction of the "Five Steps" Piano Teaching Model Focused on Students [J]. Journal of Mianyang Normal University, 2017, 36(10):82-86.

[10] Wang Chao. Application of Orff's Music Education System in Piano Teaching of Preschool Education Major in Higher Vocational Education [J]. Journal of Qiqihar Teachers College, 2017, 12(06): 113-114. 